\title{
RILEGGENDO L'EGINETICO DI ISOCRATE
}

\section{Alberto Maffi*}

1. L'Eginetico di Isocrate, una delle sei orazioni giudiziarie tramandate nel corpus dell'oratore e identificata con il $\mathrm{nr} 19$, è l'unica orazione giudiziaria attica relativa a un processo che non si è svolto ad Atene bensì ad Egina. Benché l'orazione, datata fra il 394 e il $390 \mathrm{aC}$, sollevi molte questioni di rilevanza giuridica, essa non ha suscitato un soverchio interesse presso gli storici del diritto greco. Lo studio più ampio e approfondito resta il capitolo di Hans Julius Wolff, "Der Aiginetikos des Isokrates", inserito in una Memoria del 1979 dedicata al tema del conflitto di leggi. ${ }^{1}$ Merito dello studio di Wolff è soprattutto quello di aver tentato di delineare la vicenda processuale nel suo complesso, mentre gli studi precedenti si erano concentrati quasi esclusivamente sulla questione del conflitto di leggi in base a cui valutare la validità del testamento di Trasiloco, che è in sostanza il tema al centro del processo. ${ }^{2}$ Nonostante la novità dell'approccio proposto da Wolff, non sembra che il suo lavoro abbia suscitato commenti e discussioni di particolare interesse. Mi piace quindi dedicare all'amico e collega Laurens Winkel, di cui sono noti gli importanti contributi in ambito giusgrecistico, un riesame dell'Eginetico alla luce dell'interpretazione che Wolff ne ha proposto.

1 HJ Wolff Das Problem der Konkurrenz von Rechtsordnungen in der Antike, Sitzungsberichte der Heidelberger Akad d Wissenschaften, Phil-hist K1, 1979 (5) 15-34.

2 HF Hitzig "Der griechische Fremdenprozess im Licht der neueren Inschriftenkunde" (1907) 28 ZSS 211 ss; H Lewald "Conflits de lois dans le monde grec et romain" (1959) 5 Labeo 334 ss; A Maffi "La capacità di diritto privato dei meteci nel mondo greco classico" in Studi G. Scherillo vol 1 (Milano, 1972) 177-200.

* Professore ordinario di Istituzioni di diritto romano, Università di Milano-Bicocca.

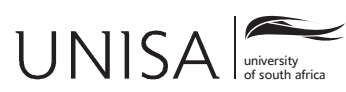


2. Prendendo spunto dal $\S$ I della trattazione di Wolff ("Parteien und Gegenstand des Prozesses"), riassumiamo la vicenda che ha dato luogo al processo. Trasillo, originario di Siphnos, aveva fatto fortuna esercitando in vari luoghi l'attività di indovino. Nel corso di queste peregrinazioni ebbe relazioni con diverse donne, alcune delle quali generarono dei figli, che, secondo l'oratore, non furono mai riconosciuti come legittimi da Trasillo ( $\$ 6)$ : fra queste donne si trovava anche colei da cui nacque quella che è l'attuale avversaria dell'anonimo che pronuncia l'orazione. Di questa donna, probabilmente rappresentata in processo da un tutore, forse suo marito, non ci viene detto né il nome né la patria. Va subito notato, a questo proposito, che l'Eginetico è un'orazione cui spetta la qualifica di amartyros, nel senso che l'oratore non suffraga mai le sue affermazioni mediante testimonianze. Dopo altre vicende matrimoniali, Trasillo ritornò a Siphnos, dove sposò una donna di Seriphos, da cui ebbe tre figli: Sopaios, Trasiloco e una figlia. Sopaios morì senza discendenti prima dei fratelli, e Trasiloco, morendo a sua volta senza discendenti, lasciò per testamento il suo patrimonio all'oratore, dandogli nel contempo in moglie la sorella.

3. Oggetto del processo è quindi l'eredità di Trasiloco, il quale, insieme all'oratore, viveva come meteco ad Egina, essendo fuggito dalla sua patria Siphnos. L'avversaria avanza una pretesa sull'eredità del fratellastro Trasiloco sulla base della comune discendenza dal medesimo padre, Trasillo (amphisbetein kata genos: $\S \S 17$, 48). L'oratore, che si trova probabilmente nel possesso dell'eredità, basa invece la sua pretesa sull'adozione testamentaria (amphisbetein kata dosin: $\S 12$, mentre al $\S$ 45 è esplicita la contrapposizione kata dosin/kata genos).

Della "figlia" di Trasillo, controparte, non viene esplicitamente ammessa o negata dall'oratore la qualità di figlia legittima di Trasillo, quindi di sorella consanguinea dei tre fratelli Sopilo, Trasiloco e della loro innominata sorella, data in moglie per disposizione testamentaria di Trasiloco all'oratore. Come abbiamo visto, l'oratore si limita ad affermare genericamente che, prima di Trasiloco e dei suoi fratelli, nessuno dei figli che Trasillo ebbe durante la sua vita errabonda fu da lui riconosciuto come legittimo. Tanto più colpisce il fatto che l'oratore non faccia leva sulla qualità di figlia illegittima della controparte, dato che, in base a questo solo motivo, alle pretese ereditarie di lei sarebbe stato tolto il fondamento giuridico.

4. Passiamo al § II della trattazione del Wolff: "Die prozessuale Lage". Il Wolff inizia rilevando giustamente che la controparte non contesta l'esistenza e la validità formale del testamento su cui l'oratore basa la sua pretesa ereditaria $(\S \S 15,34,50)$. Quel che invece vuole mettere in luce è l'invalidità sostanziale del testamento (e la conseguente richiesta ai giudici di dichiararne l'inefficacia - "Unwirksamkeit": p 17). Ciò si ricava soprattutto dal $\S 15$ : "cercano di convincervi che occorre rendere inefficace (akyrous riferito alle diathekai) il testamento." Se la controparte rivendicasse l'intera eredità o solo la metà non è specificato, ma è plausibile che 
si trattasse della metà, dato che, una volta apertasi la successione legittima, aventi diritto a succedere sarebbero state le due sorellastre.

5. Il Wolff passa quindi a interrogarsi sulla natura del processo, ossia dell'azione intentata. Si ritiene in generale che sia stata la controparte ad agire in giudizio, il che pone immediatamente il problema di spiegare come mai sia il convenuto a parlare per primo. Che sia infatti colui che pronuncia l'orazione a parlare per primo lo rivelano indizi chiari disseminati nel corso dell'orazione stessa $(\S \S 30,32,42)$. Ora, sia che si tratti di un'azione di rivendica dell'eredità (simile alla petitio hereditatis) sia che si tratti di una dike blabes, che il convenuto parli per primo si potrebbe giustificare solo se fossimo di fronte a un procedimento analogo a quello messo in moto dalla paragrafe attica. Ma una simile eventualità è da escludere. Si è parlato, in alternativa, di azione speciale o di particolarità del diritto di Egina, ${ }^{3}$ ma non ci sono elementi per suffragare simili congetture. Il Wolff (pp 18-19) ritiene quindi che si tratti di una diadikasia, il cui scopo è ottenere una sentenza di accertamento della qualità di erede. Vari elementi testuali depongono in questo senso: l'uso del verbo amphisbetein, e soprattutto di epidikazesthai ( $\$ 33 \mathrm{e} 48$ ). Il procedimento viene quindi ricostruito dal Wolff in questo modo: la controparte ha intentato l'epidikasia presso la magistratura competente di Egina, probabilmente perché lì si trovava il patrimonio ereditario (fatto di beni mobili e/o denaro). ${ }^{4}$ Ad essa si oppone l'oratore sulla base del testamento. Il motivo per cui è l'oppositore a parlare per primo potrebbe risiedere o nel fatto che è già entrato in possesso dei beni ereditari (§ 16) oppure nel fatto che, in quanto erede testamentario, appare quale "besser Berechtigte" (p 19). In ogni caso parla per primo perché è colui che ha dato il via alla diadikasia.

6. L'identificazione con una diadikasia dell'azione che ha messo in moto il processo mi sembra da condividere. Viceversa le motivazioni proposte da Wolff per spiegare il fatto che sia l'oratore a prendere per primo la parola restano alquanto opinabili, dato che, per la stessa Atene, non sappiamo chi aveva diritto di prendere la parola per primo in questo tipo di processo. Proprio il fatto che l'oratore si trovi, come sembra probabile, nel possesso dei beni ereditari, dovrebbe legittimare la controparte a pronunciare per prima il suo discorso di rivendicazione dell'eredità. Possiamo peraltro osservare che abbiamo qui una situazione molto simile a quella che i giudici di Aristofane sono chiamati a dirimere in Vespae, 583-586: tuttavia

3 G Mathieu Notice premessa all'edizione Belles Lettres (Paris, 1929) 91.

4 Si potrebbe obbiettare che di regola, almeno per quanto riguarda Atene, l'ereditiera è rivendicata tramite epidikasia dal parente che ritiene di avere titolo a sposarla, mentre nel nostro caso sembra che sia la donna stessa (sia pure assistita o rappresentata da un kyrios appartenente alla sua famiglia: $\S 4)$ a intentare l'epidikasia ( $\S \S 3$ e 48). Tuttavia se rivolgiamo di nuovo la nostra attenzione al Codice di Gortina (IC IV 72), vediamo che, anche in assenza di aventi diritto appartenenti alla famiglia, l'ereditiera avrà diritto a conseguire l'eredità in attesa di trovare un marito (col VII 40VIII 20). 
dai versi aristofanei risulta impossibile capire se sia l'erede testamentario o l'erede legittimo a parlare per primo. In ogni caso, se il tribunale assegnasse l'eredità alla controparte, ciò comporterebbe come conseguenza implicita la dichiarazione di nullità del testamento: implicita perché appare probabile che la sentenza del tribunale eginetico, analogamente a quella di un tribunale ateniese, si esprimesse soltanto attraverso un voto.

7. Un altro punto di notevole importanza, affrontato dal Wolff in questo paragrafo della sua trattazione, riguarda la questione della competenza territoriale. Come abbiamo detto, la competenza del tribunale di Egina deriverebbe dal fatto che nell'isola si trovava il patrimonio ereditario oggetto della contesa. Tuttavia si potrebbe anche avanzare l'ipotesi che la giurisdizione eginetica fosse ritenuta competente non tanto perché i beni ereditari si trovavano a Egina, quanto perché il defunto era meteco ad Egina. D'altronde lo stesso Wolff sembra adombrare questa motivazione quando, in sede di conclusioni della trattazione relativa alla Grecia delle poleis, afferma che "personen-und erbrechtliche Fragen unter Umständen nach dem Heimatrecht der betroffenen Partei beurteilt wurden" (p. 45). Al diritto della patria d'origine si potrebbe allora assimilare, qualora si tratti di meteci, il "Wohnsitzrecht", ossia il diritto del loro luogo di residenza, come avviene appunto nel caso dell'Eginetico.

8. Passiamo così al § III dell'analisi di Wolff: "Das materiellrechtliche Problem". Il punto centrale della causa consiste, come si è detto, nell'accertare la validità del testamento lasciato da Trasiloco. Se la controparte abbia le carte in regola per poter rivendicare l'eredità, cioè in sostanza se possieda effettivamente la qualità di figlia legittima del de cuius, è questione che, come abbiamo già sottolineato, l'oratore non affronta ex professo, nonostante il fatto che sembrerebbe costituire un punto fondamentale intorno a cui organizzare la sua strategia processuale. A questa singolare lacuna Wolff dedica la lunga nota 44 di pagina 20, nella quale osserva che evidentemente l'oratore riteneva inutile portare un attacco diretto allo status di figlia legittima della controparte; egli si limiterebbe quindi ad avanzare delle insinuazioni indirette, sottolineando il fatto, come si è detto, che il legame con la madre della controparte era stato solo uno fra i tanti di una lunga serie che Trasillo aveva intrecciato nel suo peregrinare in qualità di indovino per il mondo greco. Nello stesso tempo l'oratore sottolinea che Trasiloco e i suoi fratelli sarebbero gli unici figli legittimi lasciati dal defunto e da lui istituiti eredi ( $\$ \S 9$ e 43). Tutto ciò in definitiva per proteggersi dall'accusa di aver mentito apertamente se mettesse direttamente in dubbio la legittima filiazione della controparte.

9. Queste osservazioni di Wolff sono sicuramente calzanti. Tuttavia, secondo me, la questione è più complessa: occorre distinguere la questione della legittimità della nascita dalla questione della cittadinanza, che, d'altra parte, come sappiamo da Atene, sono collegate. La donna che rivendica l'eredità di Trasiloco a titolo di 
figlia (evidentemente legittima), risulta essere cittadina di una polis diversa non solo da Egina, ma anche da Siphnos. Lo dimostra il fatto che ai $\S \S 12-14$ l'oratore cita, oltre alla legge di Egina e a quella di Siphnos, anche quella del paese degli avversari (§ 14). Per stabilire allora la cittadinanza della donna occorrerebbe sapere qual era la legge sul matrimonio e sulla cittadinanza del luogo dove a Trasillo è nata questa figlia. Supponendo che la madre fosse una cittadina di quel luogo, si potrebbe pensare che, come ad Atene prima della legge di Pericle, un matrimonio con uno straniero - appunto Trasillo - fosse considerato valido, ma la figlia avrebbe acquisito la cittadinanza della madre. Quanto alla questione della filiazione legittima della donna, forse l'orazione permette di capire meglio per quale motivo l'oratore ritiene inutile, o addirittura controproducente, attaccare la controparte su questo punto. Vi sono infatti molti indizi, nella narrazione dei fatti che precedono la morte di Trasiloco, da cui risulta abbastanza chiaramente che la donna in questione, benché accusata dall'oratore di non essersi mai fatta viva nei lunghi mesi di malattia di Trasiloco e neanche al momento della sua morte, fosse tutt'altro che una persona lontana e sconosciuta, comparsa all'improvviso a vantare diritti inopinati; al contrario sembra essere stata coinvolta in varie vicende della vita dei tre figli "legittimi" di Trasiloco senza che la sua legittimazione ad avanzare pretese di carattere patrimoniale sia stata messa in discussione o abbia potuto essere efficacemente negata.

10. Ma ritorniamo al nocciolo della causa. Si chiede Wolff: come è possibile che la controparte chieda ai giudici di annullare il testamento se ella stessa ne riconosce l'esistenza e la redazione conforme a tutte le leggi di cui l'oratore dà lettura? Il carattere apparentemente paradossale di una simile domanda ha spinto alcuni autorevoli commentatori (come Blass ${ }^{5}$ e Münscher ${ }^{6}$ ) a ritenere che l'orazione non mirasse a un effettivo scopo pratico, non fosse un autentico discorso giudiziario, ma un discorso epidittico. Wolff ribadisce invece, insieme ad altri studiosi prima di lui, che si tratta di un'orazione giudiziaria redatta per un vero processo (pp 20-21). Si tratta dunque di capire per quale aspetto il testamento è considerato così manchevole o difettoso dalla controparte da chiederne ai giudici l'annullamento. La risposta di Wolff chiama in causa la legge di Solone (citata in particolare in Dem 46 c Steph 2, 14) secondo la quale è da considerarsi nullo il testamento redatto da chi non è nel pieno possesso delle sue facoltà mentali o per follia o per vecchiaia, o per effetto di droghe o farmaci, o per malattia o per l'influsso deviante di una donna. Wolff sostiene che a questa legge ateniese dovevano corrispondere norme analoghe in tutto il mondo greco. Applicando questo presupposto al caso dell'Eginetico, l'oratore si preoccuperebbe di stornare dai giudici il sospetto che Trasiloco non fosse nel pieno possesso delle sue facoltà mentali nel momento in cui ha redatto il testamento, in particolare, suggerisce Wolff, perché influenzato dalla sorella e dalla madre (con la quale ultima viene sottolineato il legame ai $\S \S 34-35$ ).

$5 \quad$ F Blass Die attische Beredsamkeit vol 2 (Leipzig, 1892).

6 Th Münscher "Isokrates" RE IX 1914. 
11. Il Wolff si rende conto che questa tesi va incontro immediatamente a un'obiezione basata sul tenore stesso dell'orazione: se il motivo della richiesta di annullamento fosse quello testé indicato, perché l'oratore non lo enuncia chiaramente e non adduce espliciti argomenti per rintuzzarlo? Wolff riconosce che l'oratore si limita a fugaci allusioni: così al § 34 si legge "[gli avversari] dicono che il testamento è stato redatto in modo sconveniente (ou kalos) e irregolare (oud'orthos)" (mentre meno pertinente mi sembra il $\S 47$, pure citato a questo proposito dal Wolff, p 23). Ma spiega questa reticenza con una misura cautelare: meglio non attirare l'attenzione dei giudici su argomentazioni della controparte che, se da lui non sottolineate, potevano risultare di minor impatto sui giudici, ai fini della decisione finale, rispetto all'immagine così negativa del comportamento della donna nei confronti della malattia e della morte di Trasiloco, che, come sottolinea Wolff, costituisce l'asse portante dell'orazione e l'argomento su cui soprattutto fa leva l'oratore. Questi si limiterebbe a contrastare indirettamente la tesi dell'invalidità del testamento, evidentemente sostenuta con forza dall'avversaria, difendendosi dalla pretesa accusa (§36) di essere un successore indegno di Trasiloco (argomento che era probabilmente presente nel discorso della controparte ma doveva trattarsi di un elemento marginale, destinato semplicemente a rafforzare la contestazione principale).

12. Ora, la ragione che il Wolff adduce, per spiegare la reticenza dell'oratore rispetto alla motivazione su cui si baserebbe la richiesta di annullamento del testamento, non mi sembra convincente. Prima di tutto il significato degli avverbi kalos e orthos del § 34, su cui il Wolff principalmente fa leva, è così vago da essere difficilmente riconducibile a un'accusa quale quella secondo lui sollevata dalla controparte. In secondo luogo, anche ammesso che ad Egina fosse in vigore una legge analoga a quella di Solone, non credo che la donna di cui la legge paventa l'influenza deviante possa essere identificata con una sorella o addirittura con la madre del testatore; anzi, proprio al $§ 34$ si elogia Trasiloco per essersi preso a cuore soprattutto la sorte della sorella, procurandole un marito, e della madre, donandole un figlio in sostituzione del defunto. Preoccuparsi del benessere della sorella e della madre rientra certamente nei doveri di un fratello e figlio: non c'è dubbio che Solone voleva impedire che un testatore avvantaggiasse ben altro genere di donna. Se proprio si volesse cercare un motivo di invalidità del testamento di Trasiloco modellato sul contenuto della legge di Solone, si potrebbe piuttosto pensare alla malattia, dato che ai $\S \S 24-27$ l'oratore insiste molto sulla gravità delle condizioni di salute di Trasiloco nei suoi ultimi mesi di vita: naturalmente lo scopo è quello di sottolineare la sua dedizione nei confronti dell'amico, accentuando per contrasto il colpevole disinteresse della controparte; tuttavia l'insistenza sullo stato di prostrazione in cui la malattia aveva ridotto Trasiloco avrebbe potuto effettivamente offrire lo spunto per sostenere che il malato non fosse più in grado di esprimere una consapevole valida 
volontà testamentaria, ${ }^{7}$ anche se la presenza dei testimoni, ${ }^{8}$ che peraltro, stando al testo che possediamo, non vengono presentati in dibattimento, doveva proprio servire a stornare questo sospetto.

13. A mio parere, però, quale che potesse essere la causa estrinseca di invalidità del testamento (influsso di una donna o malattia del testatore), non contrastare quello che, stando all'ipotesi del Wolff, costituirebbe il nucleo fondamentale dell'attacco della controparte - cioè l'accusa all'oratore di basare la propria pretesa su un testamento ispirato da una volontà deviata dall'influsso di una donna, $\mathrm{o}$, in alternativa alterata dalla malattia, sembra una scelta strategica alquanto strana, se non addirittura controproducente, perché avrebbe permesso all'avversaria di far pesare incontrastata sui giudici le argomentazioni in appoggio alla propria tesi.

14. Io credo quindi che dobbiamo andare alla ricerca di una motivazione diversa per la richiesta di annullamento del testamento di Trasiloco da parte dell'avversaria dell'oratore. A questo scopo dobbiamo prima di tutto tentare di mettere a fuoco $\mathrm{i}$ rapporti fra i personaggi coinvolti nella vicenda. Come dicevo sopra, mi sembra molto probabile che fra la donna che rivendica l'eredità e i tre figli dell'ultimo matrimonio di Trasillo già da tempo ci fossero dei rapporti alquanto stretti o quanto meno frequenti: si noti che al $\S 30$ l'oratore osserva sprezzantemente che gli avversari pretendono di adelfizein, cioè di sottolineare il legame di sangue con il de cuius, ma non contesta di fatto una simile pretesa, mentre al $\S 40$ riconosce alla controparte il titolo di sorella del defunto; si noti anche come al $\S 44$ è dato per scontato che Trasillo sia giustificato nel criticare, dall'al di là, il comportamento di sua figlia (la controparte) nei confronti degli altri tre figli. Non si spiega altrimenti che del fratello premorto di Trasiloco, Sopoli, si dica che odiava quella donna e che essa lo ostacolava o lo danneggiava (§ 17): con ciò, secondo me, l'oratore allude al fatto che, alla morte di Trasillo, la sua prima figlia, probabilmente più anziana dei tre fratelli, rivendicò e probabilmente ottenne una quota del patrimonio del padre (forse per questo era odiata, in particolare da Sopoli) e forse anche del patrimonio del fratellastro Sopoli, morto a sua volta senza figli prima di Trasiloco (§ 40). Il problema che si pone ora è se abbia diritto anche a una quota del patrimonio di Trasiloco.

15. Se Trasiloco non avesse fatto testamento, la risposta sarebbe molto probabilmente positiva: in base a un principio che è da considerarsi probabilmente panellenico, nei beni del genitore comune ai fratelli senza discendenti succedono le sorelle (v per es il Codice di Gortina: IC IV 72, V 17-22): quindi la controparte del nostro processo avrebbe avuto diritto di succedere per una quota di metà nei

7 Questa ipotesi di motivazione della pretesa invalidità del testamento di Trasiloco mi è stata suggerita dall'amico ed eminente collega Gerhard Thür nel corso di una conversazione privata.

8 come sottolinea Mathieu (n 3) 96 n 1.

9 Così già Mathieu (n 3) 95 n 2. 
beni di Trasiloco provenienti dal comune padre Trasillo. Problema diverso è se le due sorellastre sarebbero state considerate epikleroi rispetto al fratello; tuttavia, dal punto di vista patrimoniale, ossia riguardo alla parte di eredità di Trasiloco spettante a ciascuna, niente sarebbe cambiato: ciascuna avrebbe avuto diritto alla metà, fossero o meno considerate epikleroi.

16. Il problema si pone, invece, dal momento che Trasiloco ha fatto testamento nominando l'oratore erede dei propri beni attraverso il matrimonio con la sorella, ed escludendo quindi, esplicitamente o implicitamente la sorellastra. ${ }^{10} \mathrm{Mi}$ sembra infatti che ci troviamo di fronte ad un'alternativa: in un regime successorio che riconosceva la validità della successione testamentaria, si deve ritenere che un fratello privo di discendenti potesse legittimamente escludere per testamento una sorella a favore di un'altra, oppure entrambe le sorelle, discendenti dallo stesso padre, si devono ritenere epikleroi riguardo al patrimonio paterno trasmesso tramite il fratello, con la conseguenza che questi non potrebbe lasciare disposizioni testamentarie che ledono i diritti di una di loro? ${ }^{11}$

17. Il problema che si pone, in altre parole, è se una sorella possa essere considerata ereditiera rispetto a un fratello senza figli maschi, oppure se si debba applicare la disciplina dell'ereditiera solo nella successione diretta da figlia a padre. Io penso che le due parti in causa sostenessero tesi opposte. L'oratore sostiene che una sorella non si può considerare epikleros rispetto al fratello: quindi mentre si applica al padre la norma del diritto testamentario attico in base a cui il padre che abbia soltanto figlie femmine deve disporre della mano delle figlie nel testamento a favore dei designati eredi, ${ }^{12}$ lo stesso obbligo, per esclusione, non grava sul fratello, che non abbia figli, nei confronti delle proprie sorelle. Questo perché si suppone che la sorella abbia già ricevuto la sua quota di beni, o in proprietà, come a Gortina, o in forma di dote,

10 Di questa fattispecie si è occupato E Karabélias L'épiclérat attique (Athènes, 2002) 66-67. Il caso da lui esaminato, cioè la VI orazione di Iseo, mostra delle analogie ma anche delle differenze rispetto al caso di Trasiloco. La differenza più rilevante è che, mentre in Iseo l'adottante per testamento premuore al padre, nel caso dell'Eginetico il padre premuore apparentemente a tutti $\mathrm{i}$ suoi figli coinvolti nella vicenda. Quanto a Mathieu, egli dà per scontato che la sorella di Trasiloco diventi epikleros e quindi che la designazione dell'adottato come suo marito sia conforme alle regole (cita Gernet "Sur l'épiclerat" (1921) 34 REG 337-379). Si veda anche 94 n 1, dove per spiegare che gli avversari non debbono pagare deposito processuale, dice che la sorella di Trasiloco potrebbe passare per epikleros e quindi sarebbe processo per kakosis epiklerou. Ma qui allora Mathieu sembra riferirsi alla sorellastra!

11 Naturalmente l'orazione non ci dice se vi siano collaterali di Trasiloco che potrebbero rivendicare la mano delle sorellastre. Se non vi fossero, si porrebbe un ulteriore problema che nel nostro caso, essendo ignoto il diritto eginetico in materia, non potremmo comunque risolvere.

12 O quanto meno, se vogliamo adottare un'interpretazione estensiva, deve comunque lasciare loro una quota dei propri beni: $\mathrm{v}$ in proposito le opinioni contrastanti di Gagliardi e Rubinstein in Gagliardi "Per un'interpretazione della legge di Solone in materia successoria" in (2002) 5 Dike $16 \mathrm{n} 45$. 
come ad Atene. Tuttavia va osservato che nel Codice di Gortina, colonna VIII 4042 , l'ereditiera è definita colei che è priva del padre o di un fratello nato dallo stesso padre: dunque il legislatore gortinio contemplava, in via di ipotesi implicita, un caso analogo a quello in cui viene a trovarsi la donna controparte nell'Eginetico, ${ }^{13}$ che a Gortina sarebbe quindi stata considerata epikleros. Non sappiamo naturalmente quale fosse il diritto di Egina in proposito, ma mi pare si possa sostenere che l'oratore negasse alla sorella la qualifica di epikleros nei confronti del fratello. Ritengo invece che la controparte sostenesse appunto la tesi opposta: il testamento non è stato fatto né kalos né orthos perché non ha contemplato i diritti di colei che va considerata comunque ereditiera alla pari della sorella germana del testatore (si noti che sempre nel CdG, col IV 39, la divisione ereditaria tra fratelli e sorelle deve essere fatta kalos).

18. Ritornando alla posizione dell'oratore, si direbbe che adotti una doppia linea di difesa della validità del testamento. Gli avversari potrebbero sostenere, essendo a corto di altri argomenti, come dice l'oratore al $\S 42$, che Trasillo, il defunto padre della controparte, riterrebbe di subire una grave offesa vedendo sua figlia privata dei suoi beni, mentre io erediterei ciò che egli ha guadagnato. Quel che colpisce è la replica dell'oratore a questa possibile, o probabile, argomentazione avversaria: una replica tutta basata su argomentazioni retorico-giuridiche da cui è assente l'unica affermazione che ci aspetteremmo, cioè che l'avversaria non è legittimata a rivendicare l'eredità di Trasiloco. Infatti egli obietta che adesso non è il momento di occuparsi di coloro che sono morti da tanto tempo e che, d'altra parte, così come Trasillo ha lasciato i propri beni - è da supporre per testamento - a coloro che ha voluto, ${ }^{14}$ così è giusto riconoscere a Trasiloco lo stesso diritto. Questo argomento, chiaramente specioso, implica che, per l'oratore, la sua avversaria che rivendica l'eredità non debba essere considerata epikleros; e ciò proprio in base al principio, non formulato esplicitamente, che il titolare di un patrimonio, se privo di figli, può nominare per adozione un erede testamentario senza dover tener conto di eventuali pretese delle sorelle. Quindi le leggi di cui l'oratore fa dare lettura ai $\S \S 12-14$ dovevano riguardare proprio questo punto, ${ }^{15}$ e non semplicemente confermare che

13 L'Eginetico fornisce così interessanti spunti riguardo ai rapporti fra membri di famiglie aventi un solo genitore in comune: per i rapporti tra fratellastri v per es Lys $c$ Diogitone con il commento di A Damet La septième porte. Les conflits familiaux dans l'Athènes classique (Paris, 2012) 167.

14 Fra questi eredi mi pare implicito che debba essere inclusa anche la figlia ora controparte, visto che alla fine del $\S 43$ l'oratore afferma di non temere l'opinione di Trasillo e al $\S 44$ allude al fatto che Trasillo la diserederebbe se potesse vedere come si è comportata con suo figlio.

15 Il contenuto delle leggi, di cui ai $\S \S 12-14$, viene d'altronde secondo me rievocato al $\S 49$ dove non ha soltanto il valore di un luogo comune, come ritiene Mathieu (n 3) 106 n 1. In questo paragrafo l'oratore allude alla legge che consente a chi non ha figli legittimi di nominare un erede attraverso un testamento-adozione; non specifica però come sia regolato il caso in cui il testatore abbia una o più figlie femmine. 
Trasiloco aveva fatto un testamento (formalmente) regolare. Altrimenti bisognerebbe pensare che la controparte avesse impugnato il testamento sulla base del fatto che le diverse leggi prevedevano differenti requisiti di forma per la sua validità, cosa che peraltro, come si è visto, lo stesso Wolff (p 25) esclude. Wolff in realtà ritiene che la solenne citazione delle tre leggi serva ancora una volta a gettare fumo negli occhi dei giudici: convincerli di qualcosa che nemmeno gli avversari contestavano, cioè la regolarità formale del testamento, mentre in realtà era in discussione la volontà viziata del testatore, che viene invece sottaciuta dall'oratore. Ma, come abbiamo visto sopra, una motivazione di questo genere appare artificiosa ${ }^{16} \mathrm{e}$ in definitiva poco persuasiva, perché avrebbe lasciato campo libero alle argomentazioni degli avversari senza provare in alcun modo a contrastarle preventivamente.

19. Tenendo conto però del rischio che il tentativo di confermare la validità del testamento non persuadesse i giudici, in quanto convinti che la donna pretermessa dal testamento di Trasiloco fosse epikleros, all'oratore conviene sviluppare una seconda linea di difesa, insistendo sul fatto che la pretesa della controparte, pur astrattamente legittima, deve essere respinta in quanto la donna ha tenuto un comportamento che l'ha resa indegna di succedere a Trasiloco. Se questo sia soltanto un argomento morale, come ritiene Mathieu (p 97 n 1), o abbia anche una rilevanza giuridica resta incerto: si noti però, ancora una volta facendo riferimento al Codice di Gortina, che il figlio adottivo, per poter conseguire l'eredità dell'adottante, deve "adempiere gli obblighi verso gli dei e verso gli uomini” (col X 42-44).

20. Ritorniamo ora al tema del concorso o conflitto di leggi, che forma comunque uno degli aspetti più interessanti dell'orazione sotto il profilo giuridico, e che è oggetto del § IV della trattazione di Wolff ("Das Problem der anzuwendenden Rechtsnorm"). Wolff ritiene che proprio la sostanziale convergenza delle tre norme di cui viene data lettura ai $\S \S 12-14$ (il cui tenore non è purtroppo riportato nel testo dell'orazione in nostro possesso) ci impedisca di avanzare congetture su quale sarebbe stata la norma da applicare qualora esse fossero state in contrasto fra loro. In ogni caso, in mancanza di regole certe, proprie ad ogni polis o condivise a livello panellenico, per risolvere eventuali conflitti di leggi in caso di processi fra cittadini e stranieri (residenti o meno), Wolff ritiene che la soluzione dovesse basarsi sulla gnome dikaiotate (l'opinione più giusta). Per quanto riguarda poi il rilievo che la citazione delle tre leggi avrebbe potuto avere per la soluzione del caso in discussione nell'Eginetico, Wolff ritiene che la loro lettura miri in realtà a distrarre i giudici dalla reale posta in gioco: oggetto della controversia non sarebbe infatti la conformità al diritto del testamento redatto da Trasiloco, bensì l'inefficacia sostanziale delle sue disposizioni a causa della viziata formazione della volontà testamentaria: ${ }^{17}$

16 Mi sembra una petizione di principio sostenere, come fa Wolff (n 1) 26 n 54, che confermerebbe la fama di sofista già attribuita ad Isocrate addirittura da Dem 35, c Lacr, 40.

17 “... der Streit in Wahrheit ja gar nicht um die Rechtsmässigkeit der Testamenstserrichtung ... als solche ging, sondern um die etwaige materielle Unmassgeblichkeit des Akts infolge der von der Gegnerin behaupteten Unfähigkeit des Erblassers zur freien Willensbildung” (25: corsivi dell'A). 
sarebbe stato dunque per evitare di attirare l'attenzione del tribunale su quest'ultimo punto, giudicato pericoloso dall'oratore per assicurare il successo della sua tesi, che l'oratore fa appello a testi di legge che confermavano invece la validità formale del testamento.

21. Ritengo che il punto di vista di Wolff, almeno per quanto riguarda in generale il problema del diritto sostanziale da applicare alla soluzione di controversie fra cittadini e stranieri, sia plausibile, anche se da verificare in concreto: nei casi in cui fossero addotte da parte dei contendenti, appartenenti a ordinamenti diversi, leggi fra loro contrastanti, i giudici avrebbero deciso in base al loro prudente apprezzamento, quindi senza sentirsi vincolati da una delle leggi in conflitto. Nel caso dell'Eginetico l'appello dell'oratore ai giudici affinché rispettino il loro giuramento di applicare le leggi (§ 15) - apparentemente in contrasto con l'idea che non l'applicazione delle leggi ma il ricorso alla gnome dikaiotate sia il criterio a cui il tribunale dovrebbe attenersi - potrebbe essere giustificato, come sottolinea Wolff (p 25), proprio dal fatto che, stando a quel che l'oratore dichiara, le varie leggi che potrebbero essere prese in considerazione dai giudici sono fra loro concordanti. Sempre per quanto riguarda l'Eginetico, resta però il fatto, come rilevavo nel mio articolo sopra citato $(\mathrm{p}$ 183), che l'oratore riconosce, come elemento che potrebbe pesare sulla decisione dei giudici, il ricorso alla propria legge nazionale da parte dell'avversaria dell'oratore ( $\S$ 14). Ora, è vero che al $\S 15$ l'oratore sostiene che gli avversari hanno riconosciuto non solo che il testamento lasciato da Trasiloco è autentico, ma anche che tutte le leggi (relative al caso) sono a favore di colui che pronuncia l'orazione. Ritengo però che sia difficile prestare fede a un'affermazione del genere, perché, se davvero gli avversari avessero riconosciuto che le leggi applicabili al caso erano a favore della tesi dell'oratore, la loro posizione processuale ne sarebbe risultata irrimediabilmente compromessa (una volta escluso, per i motivi sopra indicati, che la pretesa della controparte si basasse su una causa invalidante la capacità di intendere e di volere di Trasiloco all'atto di redigere il testamento). Può darsi quindi che gli avversari abbiano veramente riconosciuto che le leggi che l'oratore fa leggere in dibattimento, probabilmente da lui presentate già nell'anakrisis, fossero a favore della sua tesi. Ma avranno probabilmente replicato presentando ai giudici testi di legge (magari provenienti dagli stessi ordinamenti a cui si è appellato l'oratore) che, in contrasto con quelli addotti dall'oratore, confermavano la loro tesi. Quindi, mentre l'oratore presenta testi di legge il cui contenuto sembra riguardare soprattutto la legittimità della nomina di un erede tramite adozione testamentaria da parte di chi non abbia discendenti legittimi, possiamo pensare che la controparte presentasse testi di legge da cui risultava confermato il dovere, gravante sul fratello senza discendenti legittimi, di contemplare nel testamento entrambe le sorelle epikleroi. Da questo punto di vista le tre leggi presentate nei $\S \S 12-14$, lungi dall'essere un diversivo destinato a distogliere l'attenzione dei giudici dal vero oggetto della controversia, rappresentano effettivamente il fondamento legale (sia pure parziale e funzionale al proprio scopo) su cui l'oratore basa la sua pretesa all'eredità di Trasiloco: un fondamento legale 
che viene retoricamente rafforzato dal preteso carattere panellenico della legge riguardante la successione di chi non abbia discendenti (maschi) (§ 49). Il che non esclude, come abbiamo detto, che gli avversari, pur riconoscendo la sostanziale coincidenza di contenuto dei testi di legge presentati dall'oratore, abbiano replicato portando all'attenzione del tribunale altri testi di legge, provenienti dagli stessi ordinamenti e probabilmente, ma non necessariamente, altrettanto concordanti, su cui fondare la propria contrastante pretesa. Nel caso dell'Eginetico, quindi, non è in gioco un conflitto fra leggi che regolino diversamente una medesima fattispecie, bensì la scelta fra leggi, provenienti da diversi ordinamenti, di cui le parti chiedono al tribunale l'applicazione in base all'interpretazione che darà della fattispecie oggetto della controversia. Non siamo in presenza di un conflitto di leggi, che nel caso dell'Eginetico non sembra sussistere, ma di un conflitto sulla qualificazione giuridica di una determinata fattispecie, nel nostro caso la successione di due sorelle a un fratello senza discendenti. Non siamo perciò molto lontani dal passo delle Vespe, sopra citato, dove si può immaginare che la scelta fra la prevalenza della successione kata genos rispetto alla successione kata dosin potesse trovare il suo fondamento in appropriate e specifiche previsioni legislative, questa volta all'interno del medesimo ordinamento giuridico. ${ }^{18}$

21. E' certo merito di HJ Wolff aver mostrato come i problemi di interpretazione posti dall'Eginetico di Isocrate debbano essere affrontati sulla base della comparazione con le norme in materia successoria che sono in vigore in altri ordinamenti giuridici greci. Wolff faceva riferimento soltanto al diritto ateniese, e più precisamente alla legislazione solonica. Mi pare che il confronto con le norme in materia successoria del Codice di Gortina possa dare un ulteriore contributo alla comprensione della fattispecie oggetto del processo a cui si riferisce l'Eginetico.

\section{Abstract}

Taking inspiration from the opinion of HJ Wolff, the article examines the reasons that move the adversary of the speaker to seek the annulment of Trasilochos's will. According to Wolff, the request would be in accordance with a law of Aegina analogous to the law of Solon declaring a will invalid if the testator were declared out of mind, having been accused of writing a will under the influence of a woman. But in the speech only the sister and the mother of Trasilochos are mentioned, and it was certainly not this kind of women that were considered by Solon or the supposed law of Aegina. According to the author of this article the court should rather decide whether the sisters of a brother who had died without descendants should be considered epikleroi or not. In the first case a woman who presents herself as a daughter of Trasilochos will be entitled to obtain at least half of the inheritance of her father, thus removing it from the speaker's wife.

18 Non parlerei quindi, a proposito del potere dei giudici attestato nel passo aristofaneo, di “irregularité flagrante", come fa Karabélias (n 10) 128. 